\title{
RESEARCH
}

Open Access

\section{Chronic colitis exacerbates NLRP3- dependent neuroinflammation and cognitive impairment in middle-aged brain}

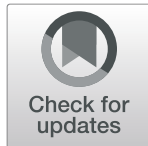

\author{
Xiao-fei He ${ }^{1 \dagger}$, Li-li Li ${ }^{1 \dagger}$, Wen-biao Xian², Ming-yue Li', Li-ying Zhang, Jing-hui Xu', Zhong Pei², \\ Hai-qing Zheng ${ }^{1 *}$ and Xi-quan $\mathrm{Hu}^{1 *}$
}

\begin{abstract}
Background: Neuroinflammation is a major driver of age-related brain degeneration and concomitant functional impairment. In patients with Alzheimer's disease, the most common form of age-related dementia, factors that enhance neuroinflammation may exacerbate disease progression, in part by impairing the glymphatic system responsible for clearance of pathogenic beta-amyloid. Inflammatory bowel diseases (IBDs) induce neuroinflammation and exacerbate cognitive impairment in the elderly. The NACHT-LRR and pyrin (PYD) domaincontaining protein 3 (NLRP3) inflammasome has been implicated in neuroinflammation. Therefore, we examined if the NLRP3 inflammasome contributes to glymphatic dysfunction and cognitive impairment in an aging mouse model of IBD.
\end{abstract}

Methods: Sixteen-month-old C57BL/6J and NLRP3 knockout (KO) mice received 1\% wt/vol dextran sodium sulfate (DSS) in drinking water to model IBD. Colitis induction was confirmed by histopathology. Exploratory behavior was examined in the open field, associative memory by the novel-object recognition and Morris water maze tests, glymphatic clearance by in vivo two-photon imaging, and neuroinflammation by immunofluorescence and western blotting detection of inflammatory markers.

Results: Administration of DSS induced colitis, impaired spatial and recognition memory, activated microglia, and increased A1-like astrocyte numbers. In addition, DSS treatment impaired glymphatic clearance, aggravated amyloid plaque accumulation, and induced neuronal loss in the cortex and hippocampus. These neurodegenerative responses were associated with increased NLRP3 inflammasome expression and accumulation of gut-derived T lymphocytes along meningeal lymphatic vessels. Conversely, NLRP3 depletion protected against cognitive dysfunction, neuroinflammation, and neurological damage induced by DSS.

Conclusions: Colitis can exacerbate age-related neuropathology, while suppression of NLRP3 inflammasome activity may protect against these deleterious effects of colitis.

Keywords: Inflammatory bowel disease, Cognition, Glymphatic clearance, NLRP3 inflammasome, T cell

\footnotetext{
*Correspondence: zhenghq2@mail.sysu.edu.cn; huxiquan@mail.sysu.edu.cn

${ }^{+}$Xiao-fei He and Li-li Li contributed equally to this work.

'Department of Rehabilitation Medicine, The Third Affiliated Hospital, Sun Yat-sen University, 600 Tianhe Road, Guangzhou 510630, Guangdong, China

Full list of author information is available at the end of the article
}

(c) The Author(s). 2021 Open Access This article is licensed under a Creative Commons Attribution 4.0 International License, which permits use, sharing, adaptation, distribution and reproduction in any medium or format, as long as you give appropriate credit to the original author(s) and the source, provide a link to the Creative Commons licence, and indicate if changes were made. The images or other third party material in this article are included in the article's Creative Commons licence, unless indicated otherwise in a credit line to the material. If material is not included in the article's Creative Commons licence and your intended use is not permitted by statutory regulation or exceeds the permitted use, you will need to obtain permission directly from the copyright holder. To view a copy of this licence, visit http://creativecommons.org/licenses/by/4.0/ The Creative Commons Public Domain Dedication waiver (http://creativecommons.org/publicdomain/zero/1.0/) applies to the data made available in this article, unless otherwise stated in a credit line to the data. 


\section{Introduction}

Neuroinflammation is strongly implicated in the pathogenesis of age-related cognitive decline, including that associated with Alzheimer's disease (AD), the most common form of dementia in older adults [1]. Patients with AD show progressive accumulation of misfolded amyloid-beta $(A \beta)$ protein within plaques, and plaque load is directly associated with the severity of neurodegeneration and eventual functional deficits. However, some plaques may be observed 10-20 years before the onset of cognitive decline [2], so there is a substantial therapeutic window for curtailing disease progression Indeed, numerous strategies have been examined to reduce $\mathrm{A} \beta$ accumulation and prevent or slow down AD progression [2], but there are currently no widely effective treatments. Growing evidence indicates that the accumulation of misfolded $A \beta$ results from an imbalance between production and clearance [3], and that impairment of $A \beta$ clearance is responsible for the most common type of $\mathrm{AD}$ [4] Thus, treatments that enhance $\mathrm{A} \beta$ clearance may be among the most broadly effective treatment strategies for AD.

The "glymphatic" pathway allows the exchange of para-arterial cerebrospinal fluid (CSF) with interstitial fluid (ISF) in the brain parenchyma, thereby promoting clearance of various toxic waste products from the central nervous system (CNS), including amyloid beta [5]. This pathway is markedly disrupted by neuroinflammation in aged brain $[6,7]$, which may lead to amyloid beta accumulation and concomitant neural damage. Glymphatic clearance is dependent on aquaporin 4 (AQP4) channels expressed at high density on the astrocytic endfeet abutting cerebral capillaries [8], but reactive astrogliosis in response to inflammatory signaling reduces AQP4 polarization [9]. Further, reactive astrocytes of the "A1" phenotype strongly expressing classical complement cascade genes are dramatically upregulated in the aged brain, contributing to neuronal death in many ageassociated neurodegenerative diseases [10, 11]. Thus, age-related neuroinflammation may impair glymphatic function.

There is also growing evidence for a regulatory role of the gut-brain axis in neuroinflammation and cognition. For example, patients with inflammatory bowel diseases (IBDs) show an elevated incidence of cognitive impairment compared to age-matched individuals without IBDs [12]. Moreover, prevention of bowel inflammation by germ-free rearing and antibiotic treatment reduces cerebral $\mathrm{A} \beta$ pathology and neuroinflammation in $\mathrm{AD}$ model mice $[13,14]$. However, it is unclear how astrocytic function and glymphatic clearance are influenced by the gut-brain axis in the elderly.

The NACHT, LRR, and pyrin (PYD) domaincontaining protein 3 (NLRP3) inflammasome is implicated in both gut immune homeostasis and neuroinflammation [15], and activation was found to exacerbate $A \beta$ deposition and cognitive impairment in $A D$ [16]. Dextran sodium sulfate (DSS)-induced colitis is the most widely used experimental animal model of IBD due to the resemblance of this condition with human IBD [17]. Ingestion of DSS induces intestinal inflammation by directly damaging the outer monolayer of colon epithelial cells, allowing intestinal contents to cross into the underlying tissue [18]. In the present study, we investigated possible contributions of the NLRP3 inflammasome to exacerbation of neurological dysfunction by DSS-induced colitis in aging mice. We found that oral administration of DSS to wild-type mice for 4 weeks increased NLRP3 inflammasome activity and gut-derived $\mathrm{T}$ cell numbers along meningeal lymphatic vessels (mLVs), induced microglial and astrocyte activation, impaired glymphatic clearance of $\mathrm{A} \beta$, and aggravated cognitive decline. In contrast, these responses were not found in NLRP3 knockout (KO) mice. Collectively, these results identify the NLRP3 inflammasome as a potential therapeutic target for AD and other neuroinflammatory disorders exacerbated by colitis.

\section{Materials and methods \\ Animals}

The study was approved by the Animal Research Committee of Laboratory Animal Monitoring Institute of Guangdong Province (Guangzhou, China; committee's reference number: [2013]97). All efforts were made to minimize the number and suffering. Both male and female mice were used in our study; NLPR3 KO mice were obtained from the Jackson laboratory (B6.129S6Nlrp3tm1Bhk/J, Catalog number: 021302) and bred in the Laboratory Animal Monitoring Institute of Guangdong Province, they were founded on a C57BL/6J background. Wild-type (WT) C57BL/6J mice were provided from the Laboratory Animal Monitoring Institute of Guangdong Province. Male animals were used at sixteen months of age and were housed under a 12:12 h light: dark cycle (light on from 07:00 to 19:00 h), with controlled temperature and humidity. WT and NLRP3 KO mice were randomly divided into two groups: Control (Ctrl) and DSS. In the control group, mice received distilled water without DSS for 28 days. According to the modified procedure as described previously [19, 20], mice in the DSS group were treated with multiple-cycle administration of $1 \% \mathrm{wt} /$ dextran sodium sulfate (DSS, molecular weight 30,000 to $50,000 \mathrm{~g} / \mathrm{mol}$, MP Biomedicals, CANADA) in drinking water on days 1 to 5,8 to 12,15 to 19 , and 22 to 26 , which was replaced by drinking water with fresh DSS solutions on day 6 to 7,13 to 14,20 to 21 , and 27 to 28 . 


\section{Open field}

Anxiety and exploratory activity were examined in the open field test [21]. The testing apparatus was a $50 \times 50$ $\mathrm{cm}$ square arena bounded by $40-\mathrm{cm}$ walls. A video camera suspended above recorded spontaneous motor activity over 5 -min trials. Mice ( $\mathrm{n}=12$ per group, six male and six female) were placed in the center of the arena, and both total distance traveled and time spent in the center (Region of interest, ROI) were recorded as indices of exploratory activity and anxiety, respectively.

\section{Morris water maze task}

Water maze tasks were performed after the finish of the DSS administration as described previously (Fig. 1A) [22, 23]. Briefly, mice ( $n=12$ per group, six male and six female) received four trials (up to $60 \mathrm{~s}$ ) on five consecutive training days, and then received a single 60 -s probe trial on day 6 . The latency to reach the platform during training days, the times crossing the target area (former platform position), and the time spent in the target quadrant during the probe trial were recorded.

\section{Novel object recognition memory test}

Recognition memory was evaluated using the novel object test as described previously [24-26]. Mice were placed in the open field arena described above and allowed to acclimate for $1 \mathrm{~h}$. Two 1-ml syringe barrels (Becton, Dickinson and Company, USA) were placed in the arena equidistant from the center, and the mouse was allowed to freely explore the environment for 10 min. Object exploration times, defined as nasal or oral contact durations, were recorded. Twenty-four hours later, mice were returned to the open field with one of the syringes replaced by a bottle cap (novel object), and contact durations with the now-familiar and novel objects recorded for $10 \mathrm{~min}$. The difference in time spent exploring the novel object versus the familiar object was calculated as an index of novel object recognition.

\section{Detection of lymphocytes migrated from the gut to the meninges}

After DSS administration, the fluorescent membrane dye CM-Dil $(5 \mu \mathrm{M}$ in $2 \mu \mathrm{L}$ of PBS per PP) (Life Technologies, USA) was injected into Peyer's patches (PPs) surrounding the ileum $[19,27]$. After two-photon imaging, mice ( $\mathrm{n}=6$ per group, three male and three female) were sacrificed and the meninges isolated for immunohistological analysis of CM-DiI labeling as described previously [28]. Briefly, mandibles and the skull rostral to the maxillae were removed, and the top of the skull with the meninges was collected and fixed in $4 \%$ paraformaldehyde (PFA) for $24 \mathrm{~h}$ at $4{ }^{\circ} \mathrm{C}$. The meninges were dissected away from the skullcap, simultaneously permeabilized with $0.3 \%$ Triton X-100 and blocked with
$10 \%$ goat serum for $1 \mathrm{~h}$ at room temperature, and then incubated overnight at $4{ }^{\circ} \mathrm{C}$ with primary rat antiNLRP3 (1:100, Thermo Fisher, USA), rabbit anti-CD3 (1:100, Abcam, USA), and rabbit anti-LYVE-1 (1:100, Abcam, USA). Immunolabeled tissues were then incubated with an Alexa Fluor ${ }^{\circ} 555$-conjugated anti-rabbit IgG $\left[(\mathrm{H}+\mathrm{L}), \mathrm{F}\left(\mathrm{ab} \mathrm{b}^{\prime}\right) 2\right.$ Fragment (1:300, Cell Signaling Technology)] and Alexa Fluor ${ }^{\circ}$ 488-conjugated anti-rat IgG $[(\mathrm{H}+\mathrm{L})(1: 300$, Cell Signaling Technology, USA)] in PBS containing $10 \%$ normal goat serum at room temperature for $1 \mathrm{~h}$. Fluorescence images were acquired using a confocal microscope (Leica, Germany).

\section{In vivo two-photon imaging of glymphatic clearance}

The efficiency of glymphatic clearance was evaluated using in vivo two-photon imaging [7]. Briefly, mice ( $\mathrm{n}=$ 6 per group, 3 male and 3 female) were anesthetized and a thin cranial window was created at the parietal. Fluorescein isothiocyanate (FITC)-dextran (70 kDa; SigmaAldrich, USA) was dissolved in artificial cerebrospinal fluid at a concentration of $1 \% ; 10 \mu \mathrm{l}$ of FITC was injected into the cisterna magna using a microsyringe connected with a syringe pump controller. $0.2 \mathrm{ml}$ of $1 \%$ rhodamine B (Sigma-Aldrich, USA) in saline was injected intravenously to show the brain vascular before imaging. Two-photon imaging on the right parietal cortex (2 mm caudal from bregma, and $1.7 \mathrm{~mm}$ lateral from the midline) was performed using a two-photon laser scanning microscope (Leica, Germany) equipped with a water immersion objective $(25 \times)$. To monitor the clearance of FITC-dextran injected into the brain parenchyma, three-dimensional (3D) xyz stacks $(512 \times 512$ pixels, $2-\mu \mathrm{m}$ resolution) were taken up to $300 \mu \mathrm{m}$ below the cortical surface at 5, 15, 30, 45, and $60 \mathrm{~min}$ after the injection of the FITC-dextran, the overall fluorescence intensities were analyzed. Besides, images $100 \mu \mathrm{m}$ below the cortical surface were obtained and the fluorescence intensities in the paravascular space were analyzed to examine the efficiency of glymphatic clearance.

\section{Histology}

Mice ( $\mathrm{n}=6$ per group, three male and three female mice) were perfused with $50 \mathrm{ml}$ ice-cold phosphate buffer saline (PBS) and $50 \mathrm{ml}$ of $4 \%(\mathrm{w} / \mathrm{v})$ paraformaldehyde in PBS. For immunofluorescence staining, coronal brain slices of right parietal cortex with $10 \mu \mathrm{m}$ thick at interval of $100 \mu \mathrm{m}$ or consecutive $40 \mu \mathrm{m}$ were sectioned. Brain sections were boiled in citric acid buffer for $5 \mathrm{~min}$ in a microwave oven and were treated with $0.3 \%$ Triton X100 and $10 \%$ goat serum for $1 \mathrm{~h}$ at room temperature and then incubated overnight at $4{ }^{\circ} \mathrm{C}$ with primary antibody [1:300 rabbit anti-ionized calcium binding adapter molecule 1 (Iba1) antibody, Wako, Japan; 1:100 rat antiNLRP3 antibody, Thermo Fisher, USA; 1:100 mouse 


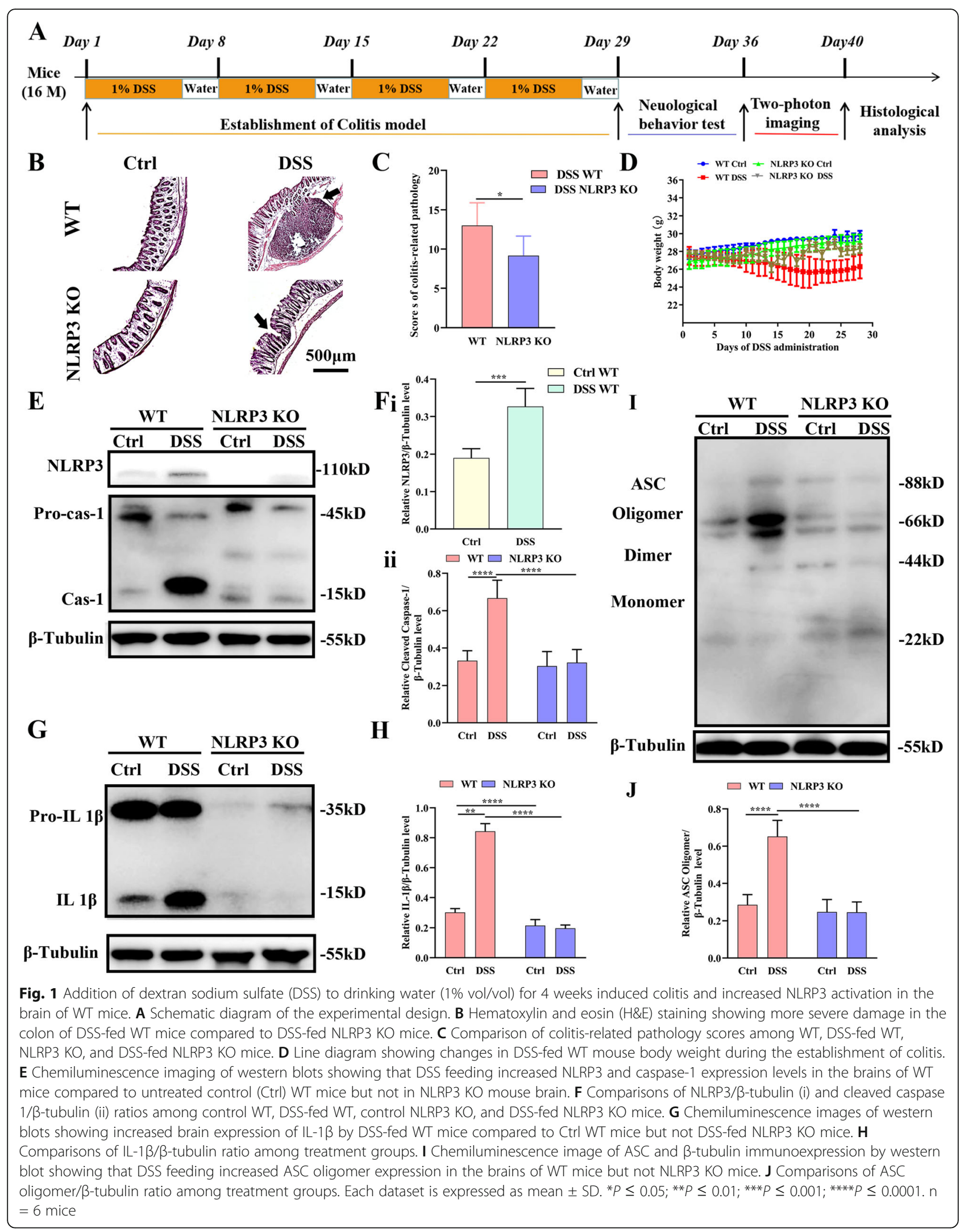


purified anti- $\beta$-amyloid, 1-42 antibody, BioLegend, USA; 1:100 mouse purified anti- $\beta$-amyloid, 1-40 antibody, BioLegend, USA; 1:300 rabbit anti-MAP2 antibody, Affility Biosciences, USA; 1:300 mouse anti-GFAP, SigmaAldrich, USA; 1:100 rabbit anti-Complement C3 antibody, Thermo Fisher, USA; 1:200 rabbit anti-AQP4, Peptide, USA; 1:300 anti-mouse NeuN, Millipore, USA] and then incubated with a secondary antibody [1:300 Anti-mouse IgG $\left.(\mathrm{H}+\mathrm{L}), \mathrm{F}(\mathrm{ab})^{\prime}\right)_{2}$ Fragment (Alexa Fluor ${ }^{\circ}$ 488 Conjugate), Cell signaling technology, USA; 1:300 Anti-rabbit IgG $(\mathrm{H}+\mathrm{L}), \mathrm{F}(\mathrm{ab}) 2$ Fragment (Alexa Fluor ${ }^{\circ}$ 555 Conjugate, Cell signaling technology, USA; 1:300 Anti-rat IgG $(\mathrm{H}+\mathrm{L})$, (Alexa Fluor 555 Conjugate), Cell signaling technology, USA] in PBS containing 10\% normal goat serum at room temperature for $1 \mathrm{~h}$. Images for $\mathrm{A} \beta$ were acquired using a Nikon fluorescence microscope (Nikon, Japan), images for other histological analysis were acquired using a confocal microscope (Leica, Germany).

\section{Grading of intestinal inflammation-related dysfunction} Body weight was monitored daily during DSS administration, and induction of colitis was confirmed by hematoxylin and eosin (H\&E) staining and histological scoring. Briefly, 2-cm pieces of the distal colon were isolated, washed in cold phosphate-buffered saline (PBS), and cut into $10-\mu \mathrm{m}$ thick sections at $100-\mu \mathrm{m}$ intervals using a freezing microtome (Leica, Germany). Sections were stained with $\mathrm{H} \& \mathrm{E}$, and colitis-related pathology graded as follows according to a previous study with modifications [29-32]: body weight loss (0, none; 1,1 $-5 \%$; $2,5-10 \% ; 3,11-15 \% ; 4,>15 \%)$ plus diarrhea severity (0, normal; 2 , loose stools; 4 , watery diarrhea) plus anal bleeding severity ( 0 , normal; 2, slight bleeding; 4, gross bleeding) plus severity of inflammation (0 none, 1 mild, 2 moderate, 3 severe) plus extent of inflammation (0 none, 1 mucosa, 2 mucosa and submucosa, 3 transmural) plus crypt damage ( 0 none, $11 / 3$ damaged, 2 2/3 damaged, 3 crypts lost, surface epithelium present, 4 crypts and surface epithelium lost). Adding the score for each parameter yielded a maximum score of 22 .

\section{Western blotting}

Six mice (three male and three female) in each group were perfused with $50 \mathrm{~mL}$ ice-cold PBS. Tissues from whole cortex and hippocampus were homogenized in $500 \mu \mathrm{L} 1 \times$ lysis buffer in a Precellys homogenizer (Stretton Scientific, Derbyshire, UK) and total protein levels were quantified using a Pierce ${ }^{\mathrm{m} m}$ Microplate BCA Protein Assay Kit (Thermo Fisher Scientific, USA) according to the manufacturer's instructions. Proteins were separated at $30 \mu \mathrm{g}$ per gel lane by sodium dodecyl sulfate polyacrylamide gel electrophoresis (SDS-PAGE) at $200 \mathrm{~V}$ for 45 min using $4 \%-12 \%$ precast polyacrylamide gels
(Novex, Invitrogen). Separated proteins were transferred to polyvinylidene fluoride membranes (Millipore, Bedford, MA, USA) at $120 \mathrm{~V}$ for $1.5 \mathrm{~h}$. Membranes were blocked in 5\% fat-free skim milk power (R\&D Systems, Minneapolis, MN, USA) for $1 \mathrm{~h}$ and incubated with the following primary antibodies overnight at $4{ }^{\circ} \mathrm{C}$ : mouse anti-NLRP3 (Thermo Fisher, USA), rabbit anti-IL-1beta (Abcam, USA) rabbit anti-caspase-1 (Abcam, USA) rabbit anti-apoptosis-associated speck (ASC, Affinity Biosciences, USA), rabbit anti-tubulin beta (Affinity Biosciences, USA), rabbit anti-beta amyloid 1-40 antibody (Abcam, USA), rabbit anti-beta amyloid 1-42 antibody (Abcam, USA), and rabbit anti-APP (Cell Signaling Technology, USA). Membranes were then incubated with secondary antibody (anti-mouse IgG, HRP-linked Antibody or anti-rabbit IgG, HRP-linked Antibody, Both from Cell Signaling Technology, USA) for $1 \mathrm{~h}$ in a dark room. Target protein bands were visualized and quantified using a chemiluminescence imaging system.

\section{Statistical analyses}

The 3D image overlays were visualized and analyzed with the Leica Application Suite (LAS) Advanced Fluorescence Lite software (LAS AF Lite, 2.4.1 build 6384, Leica, Germany). The ImageJ software (National Institutes of Health, Bethesda, MD, USA) was used to analyze the histological and western blotting results. For histological scoring of colon and NLRP3 expression, independent-samples $\mathrm{t}$ test was used to analyze. For other data, two-way repeated measures ANOVA with further turkey's multiple tests were used to analyze. A $P$ value $<0.05$ was considered statistically significant (Prism 8.0, GraphPad software, La Jolla, CA, USA). Data are expressed as means \pm standard deviations of the means (SD).

\section{Results \\ DSS administration induced colitis and activated the NLRP3 inflammasome in the brain}

We first compared histological signs of colitis, brain expression levels of the NLRP3 inflammasome, and accumulation of downstream pro-inflammatory factors between WT and NLRP3 KO mice under control conditions and following oral DSS administration for 4 weeks. Treatment groups (control and DSS-treated WT and NLRP3 KO mice) were then subjected to a battery of additional immunohistological and behavioral analyses (Fig. 1A). First, we confirmed that DSS administration induced colitis in WT mice by H\&E staining (Fig. 1B). Histological score was significantly lower in DDS-treated NLRP3 KO mice than WT mice $(\mathrm{t}=2.46, P<0.05)$ (Fig. $1 C)$, indicating that colitis was NLRP3-dependent. Furthermore, body weight was significantly reduced in DSS- 
treated WT mice but increased progressively in the other groups (Fig. 1D).

Oral DSS significantly enhanced expression of the NLRP3 inflammasome in wild-type (WT) mouse brain as evidenced by western blotting (Fig. 1E), while no NLRP3 expression was detectable in NLRP3 KO mouse brain following DSS administration $(P<0.001$ vs. WTs) (Fig. 1E and $\mathrm{F}$ i). Dual-immunofluorescence staining revealed NLRP3 inflammasomes in microglia (Supplementary figure 1A) and astrocytes (Supplementary figure 1. B) but not in neurons (Supplementary figure 1C). Furthermore, DSS administration increased cleaved caspase1 expression in the brain of WT mice $(P<0.0001)$ but not NLRP3 KO mice $(P>0.05)$, and post-DSS expression of cleaved caspase-1 was significantly lower in DSStreated NLRP3 KO mice than DSS-treated WT mice $(P$ $<0.0001$ ) (Fig. 1E and F ii).

The NLRP3 inflammasome drove the inflammatory response in part by cleaving immature interleukin (IL)- $1 \beta$ to yield the active form. In WT mice, brain expression of IL-1 $\beta$ was significantly greater following DSS treatment compared to WT controls $(P<0.0001)$, while no such change was detected in NLRP3 KO mice $(P>0.05)$, and expression was significantly lower in NLRP3 KO mice compared to WT mice following DSS $(P<0.0001)$ (Fig. 1G and H). Similarly, expression of the ASC oligomer was significantly greater in DSS-treated WT mice compared to control WT mice $(P<0.0001)$, while there was no difference in expression between control and DSS-administered NLRP3 KO mice $(P>0.05)$, and expression was significantly lower in NLRP3 KO mice compared to WT mice following DSS $(P<0.0001)$ (Fig. 1I and J). These results indicated that DSS administration activated the NLRP3 inflammasome in WT mice and increased the expression of the pro-inflammatory cytokine IL-1 $\beta$.

\section{NLRP3 depletion protected against colitis-induced neurological dysfunction}

Control and DSS-fed WT and NLRP3 KO mice were then compared for spontaneous behaviors in the open field and for cognitive functions using the Morris water maze and novel object recognition tasks. Time spent in the center of the open field (Fig. 2A), a behavioral index of anxiety, differed significantly among groups, and pairwise comparisons revealed significantly lower center time in the DSS-treated WT group, suggesting greater anxiety, compared to control WT mice $(P<0.0001)$. In contrast, there was no difference in center time between DSS-administered and control NLRP3 KO mice $(P>$ 0.05). Moreover, DSS-fed NLPR3 KO mice spent more time in the central area compared to DSS-fed WT mice $(P<0.001)$ (Fig. 2B). These increases in anxiety appear to depend on induction of colitis rather than DSS ingestion per se.

Morris water maze performance also indicated significant cognitive dysfunction in DSS-fed WT mice but not in NLPR3 KO mice. During the training phase to find a hidden platform (Fig. 2C), there were no group differences within training days (all $P>0.05$ ). However, in the probe trail for spatial memory in which the hidden platform was removed, administration of DSS significantly reduced the number of former platform crossings among DDS-fed WT mice $(P<0.01$ vs. WT controls $)$ but not NLRP3 KO mice ( $P>0.05$ vs. control KO mice), and the number of former platform location crossing was significantly greater among DSS-fed NLRP3 KO mice than DSS-fed WT mice $(P<0.01)$ (Fig. 2D and E i).Similarly, administration of DSS significantly reduced target quadrant time among WT mice compared to control WT mice $(P<0.0001)$ but had no effect on the performance of NLRP3 KO mice $(P>0.05)$, and DSS-fed NKRP3 KO mice spent more time in the target quadrant than DSSfed WT mice $(P<0.0001)$ (Fig. 2D and E ii). Collectively, these findings suggest that colitis induces a NLRP3 inflammasome-dependent spatial memory deficit.

Further, recognition memory was also impaired in DSS-fed WT mice but not DSS-fed NLRP3 KO mice as evidenced by the novel object recognition test [25]. As expected, control WT, control NLRP3 KO, and DSS NLRP3 KO groups spent more time in contact with the novel object than the previously presented (familiar) object $(P<0.01, P<0.0001$, and $P<0.001$, respectively $)$ (Fig. 2F and G i). However, DSS-fed WT mice spent less time in contact with the novel object than the familiar object $(P<0.01)$, while time spent in contact with the familiar object did not differ significantly among the other treatment groups (all $P>0.05$ ). Treatment of WT mice with DSS significantly reduced the time contacting the novel object $(P<0.0001)$ but DSS had no effect on NLRP3 KO mice $(P>0.05)$, and time contacting the novel object was significant higher among DSS-fed NLRP3 KO mice than DSS-fed WT mice $(P<0.0001)$ (Fig. 2F and G).

\section{NLRP3 depletion inhibited colitis-induced microglial activation and protected against neuronal loss}

We then analyzed the effect of DSS on neuronal survival and the potential protection conferred by NLRP3 depletion. Consistent with the cognitive dysfunction induced by DSS in WT but not NLRP3 mice, cortical neuron number was significantly lower in DSS-fed WT mice than control WT mice $(P<0.05)$ while there was no difference between DSS-fed and control NLRP3 KO mice $(P>0.05)$. The number of cortical neurons was also greater in DSS-fed NLRP3 KO mice than DSS-fed WT group $(P<0.05)$ (Fig. $3 \mathrm{~A}$ and $\mathrm{Bi})$. In contrast, the 


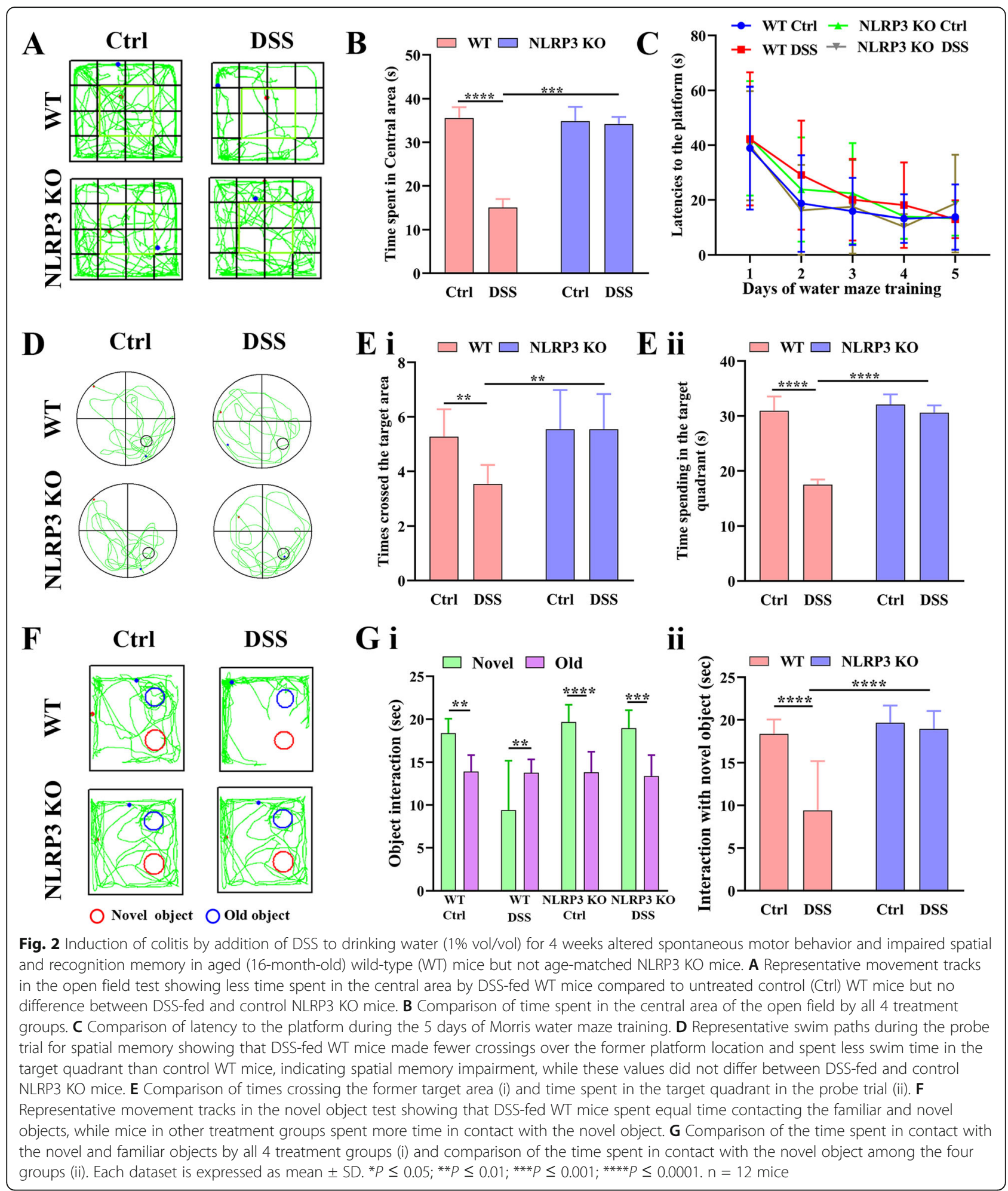

number of cortical microglia was significantly higher in DSS-fed WT mice compared to control WT mice $(P<$ $0.001)$ while there was no difference between DSS-fed and control NLRP3 KO mice $(P>0.05)$. The number of cortical microglia was also lower in DSS-fed NLRP3 KO mice than DSS-fed WT mice $(P<0.001)$ (Fig. $3 \mathrm{~A}$ and $\mathrm{B}$ ii), suggesting the suppression of NLRP3 inflammasome activity protected against colitis-induced neuroinflammation and neurodegeneration. In the hippocampus as well, hippocampal neuron number was significantly 


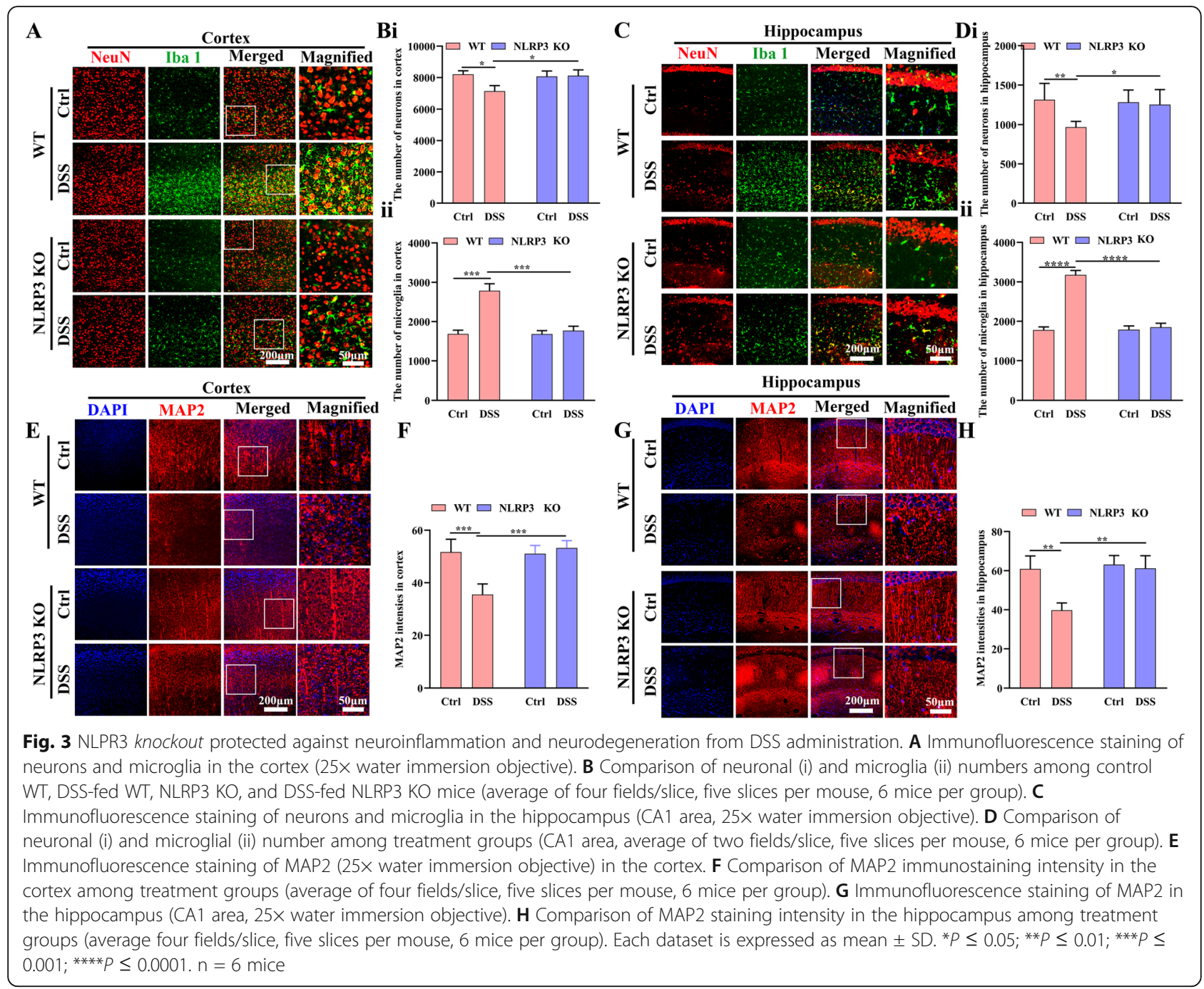

lower in DSS-fed WT mice than control WT mice $(P<$ 0.01 ) but did not differ between DSS-fed and control NLRP3 KO mice $(P>0.05)$, and hippocampal neuron number was significantly higher in DSS-fed NLRP3 KO mice than DSS-fed WT mice $(P<0.05)$ (Fig. $3 C$ and D i). Similar to the cortex, the number of microglia was significantly higher in the hippocampus of DSS-fed WT mice compared to control WT mice $(P<0.0001)$ but did not differ between DSS-fed and control NLRP3 KO mice $(P>0.05)$ and was significantly lower in DSS-fed NLRP3 KO mice than DSS-fed WT group $(P<0.0001)$ (Fig. 3C and $D$ ii). These results indicated that DSS administration induced neuronal loss in the hippocampus and that this neurodegenerative response was dependent on NLRP3.

We also quantified neuronal functional integrity by immunostaining for MAP2 in the cortex and hippocampus. Mean MAP2 expression intensity was lower in the cortex of DSS-fed WT mice than control WT mice $(P<$ 0.001), but did not differ between control and DSS-fed
NLRP3 KO mice $(P>0.05)$. Cortical MAP2 expression was also significantly higher in DSS-fed NLRP3 KO mice than DSS-fed WT mice $(P<0.001)$ (Fig. 3E and F). In the hippocampus as well (Fig. 3G and H), MAP2 expression intensity was significantly lower in DSS-fed WT mice than control WT mice $(P<0.01)$ but did not differ between DSS-fed and control NLRP3 KO mice $(P>$ $0.05)$ was significant greater in DSS-fed NLRP3 KO mice than DSS-fed WT mice $(P<0.01)$.

\section{NLRP3 depletion attenuated the colitis-induced amyloid beta deposition}

Among individuals destined to develop age-related mild cognitive impairment (MCI) and $\mathrm{AD}$, neuronal loss and neuroinflammation is associated with accumulation of $A \beta$, so we analyzed the effect of DSS administration on deposition of pathogenic $A \beta(A \beta 1-40, A \beta 1-42)$ and the potential protective efficacy of NLRP3 depletion (Fig. 4). Consistent with cognitive and histological evaluations, administration of DSS significantly increased A $\beta 1-40$ 


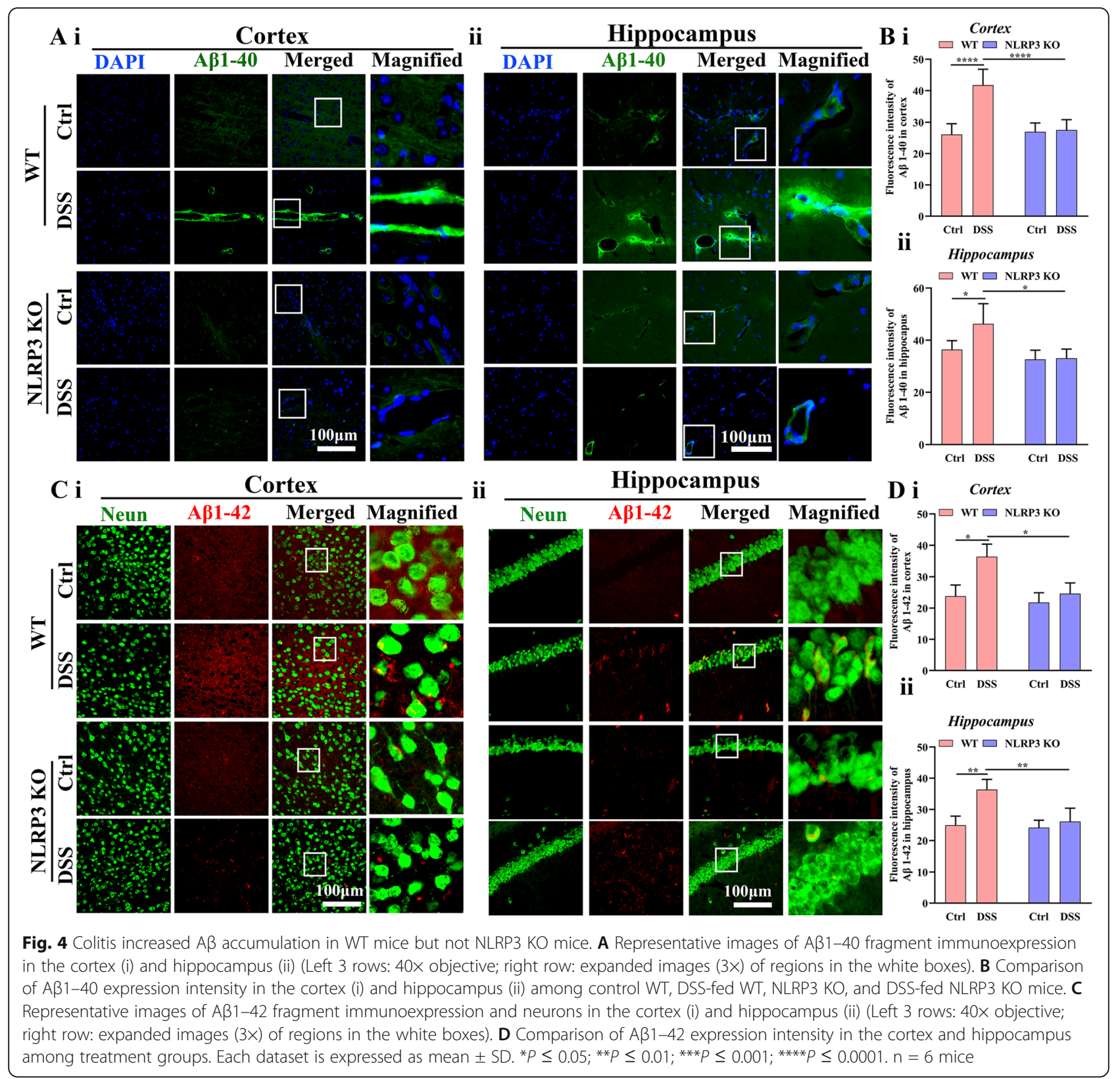

expression in the cortex of WT mice $(P<0.0001)$ but not NLRP3 KO mice $(P>0.05)$, and cortical A $\beta 1-40$ expression was significantly lower in DSS-fed NLRP3 KO mice then DSS-fed WT mice $(P<0.0001)$ (Fig. 4A i and $\mathrm{B}$ i). In the hippocampus as well (Fig. 4A ii and B ii), DSS administration significantly increased A $\beta 1-40$ deposition in WT mice $(P<0.05)$ but not NLRP3 KO mice $(P>0.05)$, and A $\beta 1-40$ expression was significantly lower in DSS-fed NLRP3 KO mice than DSS-fed WT mice $(P<0.05)$. Similarly, administration of DSS also significantly increased A $\beta 1-42$ deposition in the cortex of WT mice $(P<0.05)$ but not NLRP3 KO mice $(P>$ $0.05)$, and $A \beta 1-42$ expression was significantly lower in
DSS-fed NLRP3 KO mice than DSS-fed WT mice $(P<$ 0.05 ) (Fig. 4C i and D i), DSS administration significantly increased hippocampal A $\beta 1-42$ deposition in WT mice $(P<0.01)$ but not NLRP3 KO mice $(P>0.05)$, while A $31-42$ intensity was significantly lower in DSS-fed NLRP3 KO mice than DSS-fed WT mice $(P<0.05)$ (Fig. $4 C$ ii and D ii). We also measured amyloid precursor protein (APP) and A $\beta$ fragments (Supplementary Fig. 2) by western blotting. There were no significant pair-wise differences in APP expression levels among the four treatment groups (all $P>0.05$ ) (Supplementary Fig. $2 \mathrm{~A}$ and $B$ i), while both A $\beta 1-40$ (Supplementary Fig. 2 A and $B$ ii) and A $\beta 1-42$ (Supplementary Fig. 2 C and D) 
expression levels were higher in DSS-fed WT mice than control WT mice $(P<0.0001, P<0.05)$ but did not differ between DSS-fed and control NLRP3 KO mice (both $P>0.05)$. Also, $A \beta 1-40$ and $A \beta 1-42$ expression levels were lower in DSS-fed NLRP3 KO mice than DSS-fed WT mice $(P<0.0001, P<0.01)$. Thus, colitis induced NLRP3-dependent $A \beta$ accumulation in the cortex and hippocampus, possibly by suppressing glymphatic clearance, consistent with the observed neuropathology, neuroinflammation, and impaired spatial cognition.

\section{NLRP3 depletion decreased astrocytic activation induced by colitis}

We then examined the effects of colitis and NLRP3 $\mathrm{KO}$ on astrocyte density and polarization of astrocytic AQP4 surface expression in the cortex and hippocampus as measures of astrocytic activation and function. In the cortex (Fig. 5A), the astrocyte density was higher in DSS-fed WT mice compared to control WT mice $(P<0.0001)$ but did not differ between DSS-fed and control NLRP3 KO mice $(P>0.05)$ and was significantly lower in DSS-fed NLRP3 KO mice than DSS-fed WT mice $(P<0.0001)$ (Fig. 5B i), suggesting that NLRP3 KO protected against neuroinflammation and reactive transformation of astrocytes. There were no significant pair-wise differences in AQP4 immunoexpression intensity among the four treatment groups (all $P>0.05$ ) (Fig. 5B ii). Alternatively, the AQP4 polarity was significantly lower in DSS-fed WT mice than in control WT mice $(P<0.001)$. Conversely, there was no difference in polarity between DSS-fed and control NLRP3 KO mice $(P>0.05)$. Polarity was significantly higher in DSS-fed NLRP3 KO mice compared to DSS-fed WT mice $(P<0.001)$ (Fig. $5 \mathrm{~B}$ iii).

In the hippocampus as well (Fig. $5 \mathrm{C}$ and D), astrocyte density was significantly higher in DSS-fed WT mice compared to control WT mice $(P<0.0001)$, but not significantly different between DSS-fed and control NLRP3 KO mice $(P>0.05)$. The density of hippocampal astrocytes was significantly lower in DSS-fed NLRP3 KO mice than DSS-fed WT mice $(P<0.0001)$ (Fig. 5D i). There were no pair-wise differences in hippocampal AQP4 expression among the four groups (all $P>0.05$ ) (Fig. 5D ii). The polarity of AQP4 in the hippocampus was also significantly lower in DSS-fed WT mice than control WT mice $(P<0.001)$ but did not differ between DSS-fed and control NLRP3 KO mice $(P>0.05)$ and was significantly greater in DSS-fed NLRP3 KO mice than DSS-fed WT mice $(P<0.05)$ (Fig. 5D iii). These findings suggest that DSS induces neuroinflammation and impairs astrocytic function through enhanced NLRP3 inflammasome activity.

\section{NLRP3 depletion decreased the elevation in A1-like} astrocyte numbers induced by colitis

We investigated the effect of DSS administration on astrocyte phenotype by GAFP and C3 immunofluorescence staining (Fig. 6). Administration of DSS significantly increased cortical C3 expression in WT mice $(P<$ $0.05)$ but not NLRP3 KO mice $(P>0.05)$, and cortical C3 expression was significantly lower in DSS-fed NLRP3 KO mice than DSS-fed WT mice $(P<0.05)$ (Fig. $6 \mathrm{~A}$ and B i). Similarly, administration of DSS increased C3positive astrocyte numbers in the cortex of WT mice $(P$ $<0.001)$ but not NLRP3 KO mice $(P>0.05)$, and C3positive astrocyte number was significantly lower in the cortex of DSS-fed NLRP3 KO mice compared to DSSfed WT mice $(P<0.01)$ (Fig. 6A and B ii). In the hippocampus as well, $\mathrm{C} 3$ expression was significantly higher in DSS-fed WT than control WT mice $(P<0.05)$ but did not differ between DSS-fed and control NLRP3 KO mice $(P>0.05)$ and was also significantly lower in DSSfed NLRP3 KO mice than DSS-fed WT mice $(P<0.05)$ (Fig. 6C and D i). Consistent with $\mathrm{C} 3$ expression levels, the number of C3-positive astrocytes in the hippocampus was significantly higher in DSS-fed mice than control WT mice $(P<0.001)$ but did not differ between DSS-fed and control NLRP3 KO mice $(P>0.05)$. The number of C3-positive hippocampal astrocytes was also significantly lower in DSS-fed NLRP3 KO mice than DSS-fed WT mice $(P<0.001)$ (Fig. $6 \mathrm{C}$ and D ii). These results indicated that DSS administration promoted the A1-like transformation of astrocytes and that this effect was dependent on NLRP3 activation.

\section{NLRP3 depletion rescued the impairment in glymphatic clearance induced by colitis}

To directly examine the effect of colitis on glymphatic function, we measured FITC-dextran clearance in vivo using two-photon imaging (Fig. 7). Following intracisternal injection, the FITC tracer moved along the cerebral vasculature and entered the cortical parenchyma. Threedimensional analysis revealed a progressive rise in FITC fluorescence intensity for $30 \mathrm{~min}$ before decreasing in WT control and both NLRP3 groups, while the tracer signal continued to increase for $60 \mathrm{~min}$ in DSS-fed WT mice, suggesting impaired glymphatic clearance (Fig. 7A and $\mathrm{B}$ i). There were no significant pair-wise differences among groups (all $P>0.05$ ) at $5 \mathrm{~min}$ after tracer injection (Fig. 7A and B ii). At $60 \mathrm{~min}$ after tracer injection, however, tracer fluorescence intensity was significantly greater in DSS-fed than control WT mice $(P<0.001)$ but did not differ between NLRP3 KO groups $(P>0.05)$, and was significantly lower in DSS-fed NLRP3 KO mice than DSS-fed WT mice $(P<0.0001)$ (Fig. 7A and B iii).

To examine the efficiency of interstitial fluid flow, we then analyzed FITC-dextran movement in the 


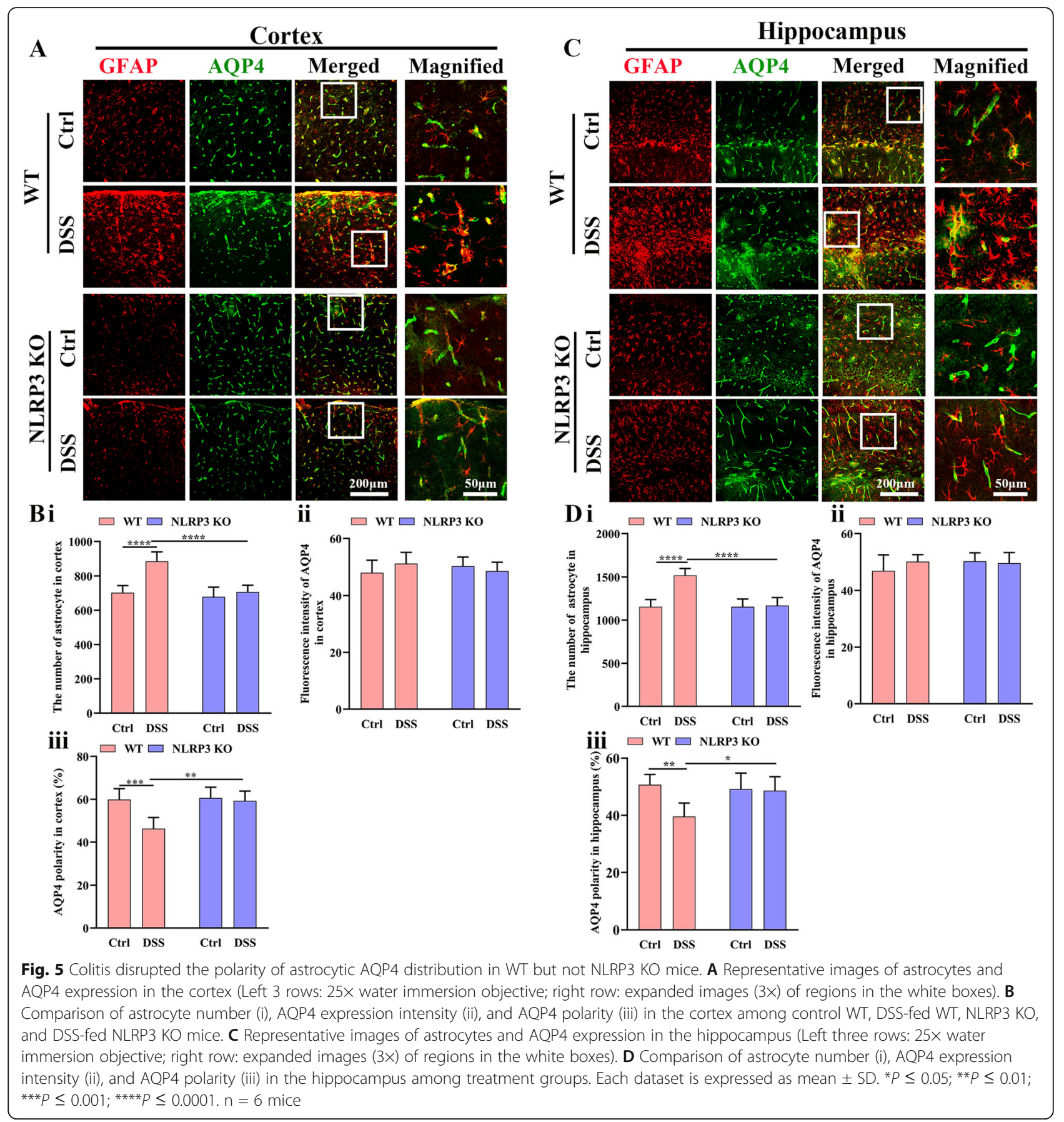

paravascular space at $100 \mu \mathrm{m}$ below the cortical surface (Fig. 7C and D). In DSS-fed WT mice, FITC signal intensity kept increasing from 5 to 45 min postinjection, and then gradually decreased, while all other groups demonstrated decreases in signal intensity starting $5 \mathrm{~min}$ following injection (Fig. $7 \mathrm{C}$ and D i). Specifically, at $5 \mathrm{~min}$ following injection, there were no significant differences in FITC intensity among the four groups at $5 \mathrm{~min}$ post-injection (all $P$ $>0.05$ ) (Fig. 7C and D ii). At $60 \mathrm{~min}$ following FITC- dextran injection, however, FITC intensity was significantly higher in DSS-fed WT mice than control WT mice $(P<0.001)$ but did not differ between NLRP3 KO groups $(P>0.05)$ and was significantly lower in DSS-fed NLRP3 KO mice compared to DSS-fed WT mice $(P<0.001)$ (Fig. $7 \mathrm{C}$ and $\mathrm{D}$ iii). Collectively, these results indicated that DSS-induced colitis and neural NLRP3 inflammasome activity impaired glymphatic clearance, while NLRP3 inflammasome suppression mitigated this effect. 


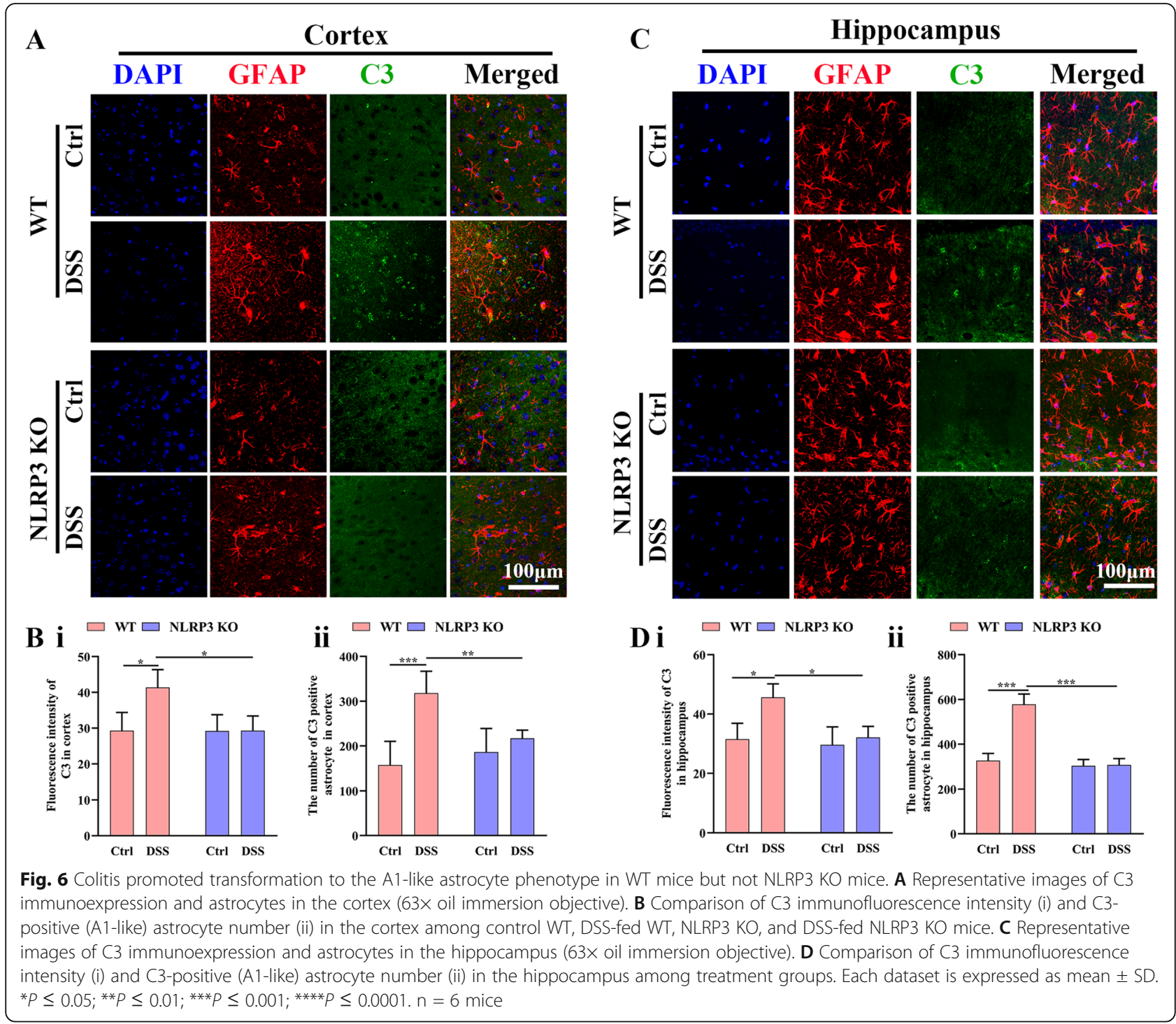

NLRP3 depletion inhibited meningeal accumulation of gut-derived $\mathrm{T}$ cells

A previous study reported that the neuroinflammation associated with colitis was enhanced by migration of activated $\gamma \delta \mathrm{T}$ cells from the gut to the meninges [33], so we examined if the differences in neuroinflammation and functional deficits among groups reflected meningeal gut-derived $\mathrm{T}$ cell accumulation (Fig. 8). There were a significantly greater number of CD3-positive cells in the mLVs of DSSfed WT mice than control WT mice $(P<0.0001)$ but not in the mLVs of DSS-fed NLRP3 KO mice compared to control NLRP3 KO mice $(P>0.05)$ (Fig. 8A and $\mathrm{C} \mathrm{i}$ ). In addition, there were a significantly greater number of CM-Dil-positive cells in the mLVs of DSS-fed WT mice than control WT mice $(P<0.0001)$ but not in the mLVs of DSS-fed NLRP3 KO mice compared to control NLRP3 KO mice $(P>$
0.05) (Fig. 8B and C ii). Moreover, CM-Dil-positive cell number was significantly lower in the mLVs of DSS-fed NLRP3 KO mice compared to DSS-fed WT mice $(P<0.0001)$ (Fig. $8 \mathrm{~B}$ and $\mathrm{C}$ ii). It was previously confirmed that CM-Dil microinjection into PPs only labels cells located within PPs without systemic cell labeling (i.e., in blood or spleen) [27]. Therefore, these findings suggest that recruitment of gutderived lymphocytes to the meninges is dependent on NLRP3 inflammasome activity. There was no obvious NLRP3 inflammasome activity in the meninges of NLRP3 KO mice, while DDS feeding significantly enhanced NLRP3 inflammasome activity in WT mice ( $\mathrm{t}=6.68, P<0.0001$ ) (Fig. $8 \mathrm{D}$ and $\mathrm{E} \mathrm{i}$ ), but for expressions of the lymphatic endothelial cell marker, there was no difference in LYVE-1 intensity among groups (all $P>0.05$ ) (Fig. $8 \mathrm{D}$ and $\mathrm{E}$ ii). 


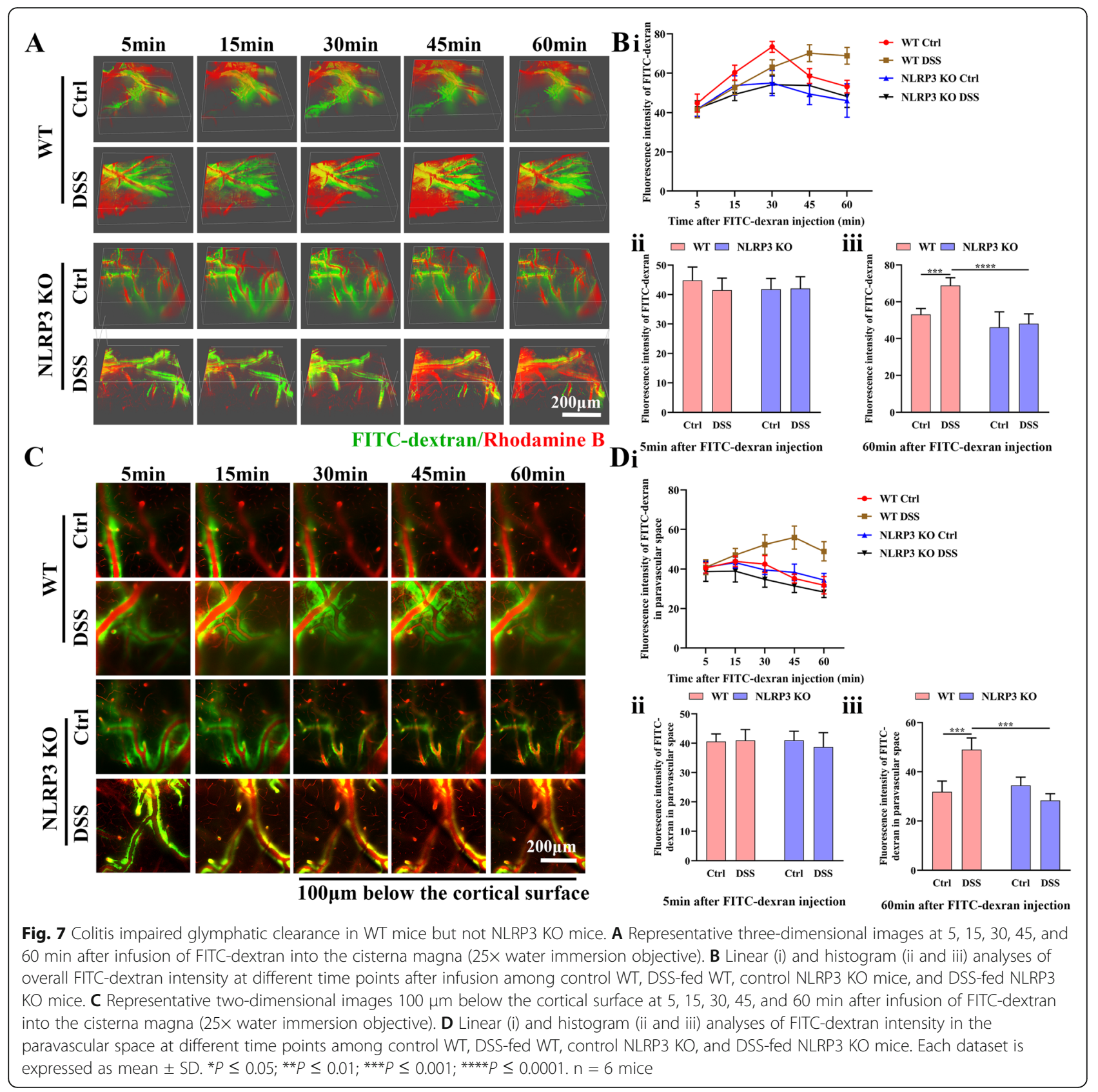

\section{Discussion}

There is compelling evidence that the gut-brain axis (GBA) regulates the progression of neurodegenerative diseases, including $\mathrm{AD}[34,35]$, and that intestinal lesions even decades before AD diagnosis may accelerate the underlying neuropathological processes [2]. Consistent with this notion that intestinal lesions can exacerbate early AD-like pathology, intestinal inflammation induced by DSS disrupted glymphatic clearance, increased $A \beta$ deposition, triggered neuroinflammation, impaired spatial cognition, and induced anxiety-like behavior among aging mice. Furthermore, these pathological effects were dependent on the NLRP3 inflammasome as all were suppressed by NLRP3 knockout, underscoring the therapeutic potential NLRP3 inflammasome blockade for early treatment of $\mathrm{AD}$.

Individuals with IBD are reported to be at increased risk of developing anxiety or depression [36], and patients with a history of anxiety or depression are at increased risk of dementia and cognitive impairment [3739]. Consistent with a mediating role for anxiety in colitis-associated cognitive impairment, anxiety-like behavior in the open field was accompanied by impaired spatial memory during the MWM probe trial and with 


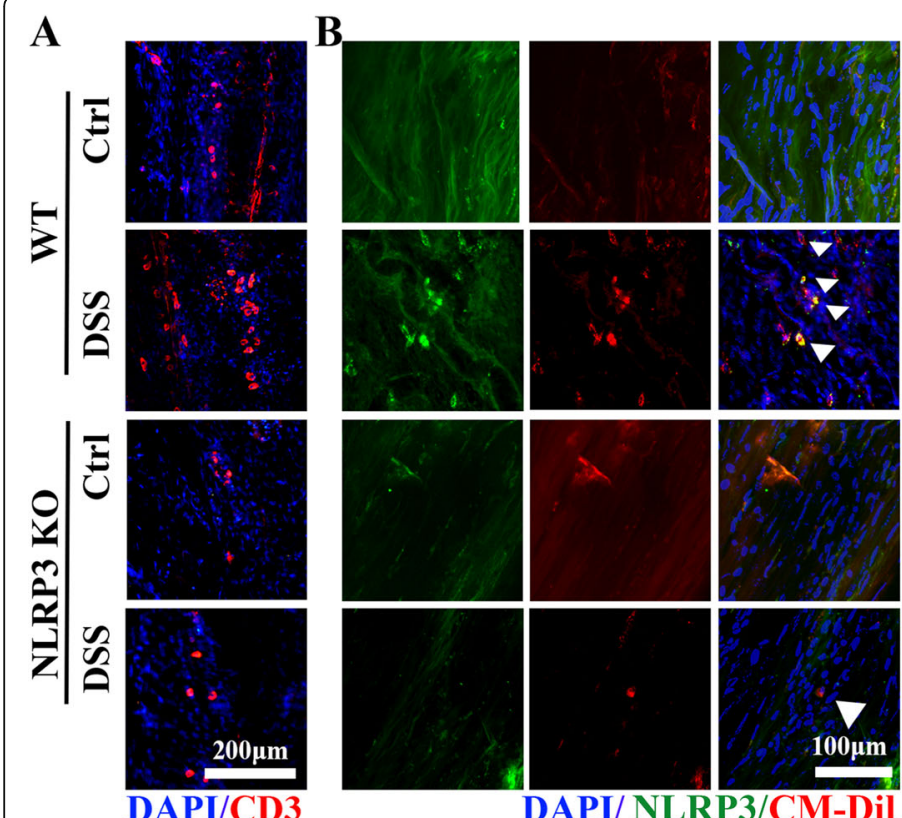

Ci $\square$ wT $\square$ NLRP3 Ko

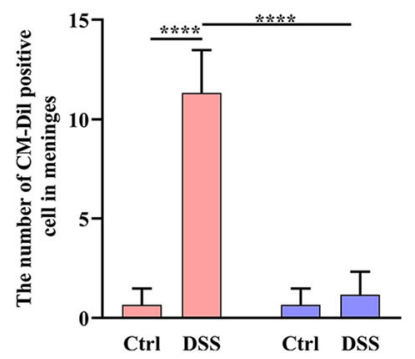

ii $\square$ WT $\square$ NLRP3 Ko

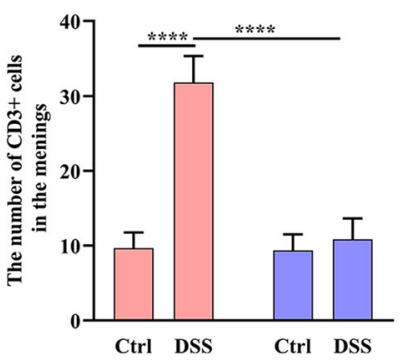

D

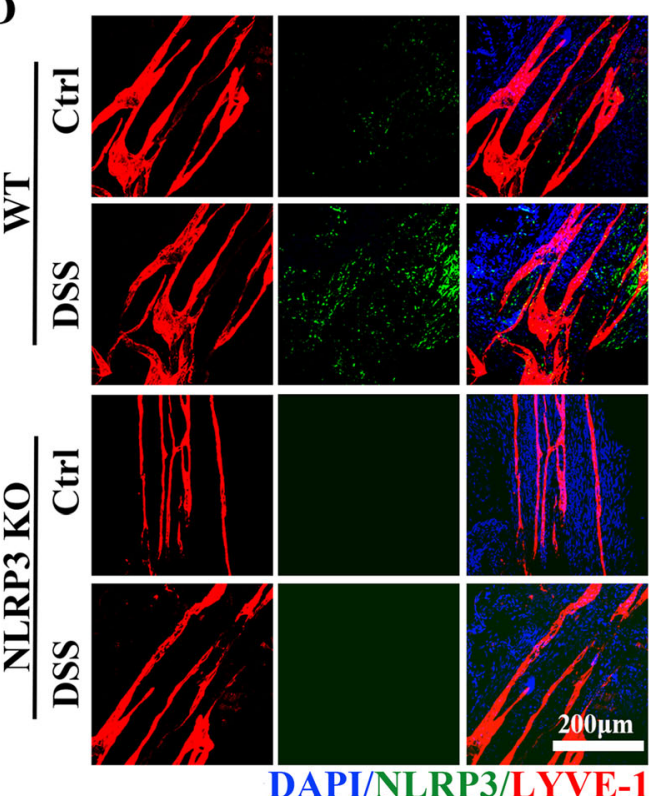

$\mathbf{E} \mathbf{i}$

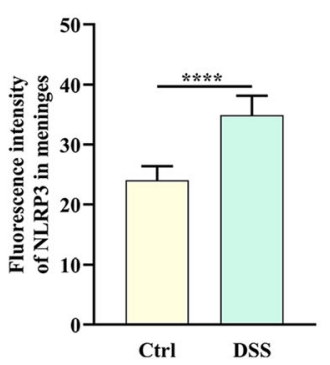

ii $\square$ wT $\square$ NLRP3 Ko

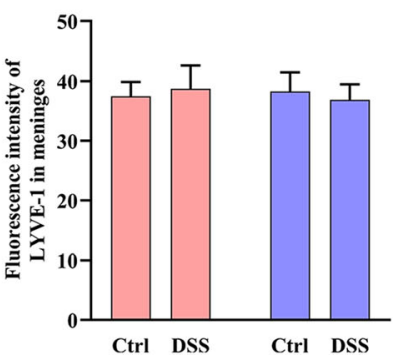

Fig. 8 Colitis increased NLRP3 inflammasome expression and accumulation of gut-derived cells in the meninges of WT mice but not NLRP3 KO mice. A Representative images of CD3 immunoexpression in the meninges (25x water immersion objective). B Representative images of CM-Dilpositive (gut-derived) cells and NLRP3 expression in the meninges (25x water immersion objective, magnified 3x). C Comparison of CD3-positive cell number (i) and CM-Dil-positive cell number (ii) in the meninges of WT and NLRP3 KO mice. D Representative images of NLRP3 inflammasome and LYVE-1 immunoexpression in the meninges (25× water immersion objective). E Comparison of NLRP3 inflammasome (i) and LYVE1 intensities (ii) in the meninges of WT and NLRP3 KO mice. Each dataset is expressed as mean \pm SD. ${ }^{*} P \leq 0.05 ;{ }^{* *} P \leq 0.01 ;{ }^{* * *} P \leq 0.001$; **** $P \leq 0.0001 . n=6$ mice

reduced novel object recognition. Normal animals exhibit a preference to explore novel objects over familiar objects, so reduced attention or interaction with novel objects is a sign of recognition memory dysfunction [25]. In contrast, DSS-induced colitis did not affect the escape latencies during MWM training, consistent with a previous study reporting that colitis impairs spatial memory without influencing spatial learning [40].

Microglial activation by the NLRP3 inflammasome may be a critical contributor to increased $A \beta$ deposition. First, microglia are the resident macrophages and primary immune cells in the central nervous system responsible for the phagocytosis and clearance of $A \beta$ [41] and NLRP3 activation was found to promote proinflammatory responses of microglia and dampen $A \beta$ clearance [42-44]. Moreover, astrocytes are also responsible for $A \beta$ clearance [45], and microglial activation induces A1-like astrocyte transition, resulting in a functionally deficient phenotype $[10,11]$. Consistent with these findings, DSS-induced colitis increased microglial activation and A1-like astrocyte numbers, and these responses were inhibited by NLRP3 depletion.

Dysfunction of glymphatic clearance has been shown to enhance $A \beta$ deposition [46], and indeed, DSS-fed WT mice showed greater $A \beta$ accumulation concomitant with reduced glymphatic clearance of a dextran tracer as well as multiple additional signs of glymphatic impairment such as loss of AQP4 polarity. As a lymphatic-like system in the brain, the glymphatic pathway drains protein wastes to the cervical lymphatics both in humans and mice $[8,47]$. Surface expression of AQP4 must be polarized to astrocytic endfeet abutting cerebral vessels for 
efficient glymphatic clearance [10, 48], and the reactive astrocytes from DSS-fed WT mice demonstrated reduced AQP4 polarity. The pro-inflammatory cytokine IL-1 $\beta$ is considered a key mediator of NLRP3-induced glymphatic dysfunction. First, NLRP3 triggers the maturation of IL-1 $\beta$, which binds to cognate receptors on astrocytes leading to astrogliosis [49] and loss of AQP4 polarity, while depletion of NLRP3 decreases mature IL$1 \beta$ production and improves AQP4 polarity. Mature IL$1 \beta$ binding to IL-1 receptors was reported to upregulate the expression of pro-IL-1 $\beta$ [50]. Moreover, reactive A1like astrocytes in the aged brain produce an exaggerated response to $\mathrm{IL}-1 \beta$ [11]. In addition to $A \beta$, glymphatic dysfunction may also lead to enhanced formation of neurofibrillary tangles. Tau protein can be transferred between cells through the fluid in extracellular space, so glymphatic dysfunction may exacerbate neuron-toneuron propagation [8], a process that will be explored in our future studies.

Accumulation of gut-derived $\mathrm{T}$ cells in mLVs is another potential contributor to age-related neuropathology and cognitive decline [33]. Meningeal LVs alter the accessibility of immune neuromodulators to the brain parenchyma, thereby potentially exacerbating inflammation [51]. In addition, CD4 $+\mathrm{T}$ cells in the meninges were reported to enter the CSF [52], induce microglial activation, and enhance local pro-inflammatory cytokine production $[33,53,54]$. Using in vivo CM-Dil cell tracing, we found that $\mathrm{CD} 4+\mathrm{T}$ cells in the paracolic lymph nodes migrated to the meninges. Furthermore, brain cytokines have been demonstrated to promote $\mathrm{T}$ cell infiltration [19]. The reduced neuropathology observed in NLRP3 KO mice following DSS-induced colitis may have resulted from lower mature IL-1 $\beta$ production and ensuing infiltration of fewer gut-derived $\mathrm{T}$ cells.

Dysfunction of mLVs due to DSS treatment may also contribute to $A \beta$ plaque accumulation [55]. However, LYVE-1 staining indicated that meningeal lymphatic endothelial cells were unaffected by colitis or NLRP3 depletion. Indeed, mLVs express a unique transcriptional signature, and meningeal lymphatic endothelial cells do not undergo expansion during inflammation $[55,56]$. There is another limitation in this study, which will be explored in the future. We cannot exclude the possibility that the protection conferred by NLRP3 depletion was due to attenuation of experimental colitis $[57,58]$ as depletion also decreased the histological colitis score.

\section{Conclusion}

We demonstrate that intestinal inflammation can trigger neuroinflammation and decreased glymphatic clearance efficacy in aging mice, resulting in increased $A \beta$ deposition and ultimately in neuronal death and cognitive impairment. We further demonstrate that these effects are likely mediated in part by migration of gut-derived $\mathrm{CD} 4+\mathrm{T}$ cells and activation of the NLRP3 inflammasome. These mechanisms linking gut to brain inflammation are potential therapeutic targets for the treatment of neurodegenerative diseases such as AD.

\section{Abbreviations}

IBDs: Inflammatory bowel diseases; NLRP3: NACHT-LRR and pyrin (PYD) domain-containing protein 3; DSS: Dextran sodium sulfate; A $\beta$ : Amyloid-beta; AD: Alzheimer's disease; mLVs: Meningeal lymphatic vessels; KO: Knockout; IL$1 \beta$ : Interleukin-1ß; ASC: Apoptosis-associated speck; MAP2: Microtubuleassociated protein 2; ANOVA: Analysis of variance; AQP4: Aquaporin-4; GAFP: Glial fibrillary acidic protein; Iba1: Ionized calcium-binding adapter molecule 1; FITC: Fluorescein isothiocyanate; GBA: Gut-brain axis;

CSF: Cerebrospinal fluid; ISF: Interstitial fluid

\section{Supplementary Information}

The online version contains supplementary material available at https://doi. org/10.1186/s12974-021-02199-8.

Additional file 1:. Supplementary figure 1. NLRP3 inflammasome upregulation induced by colitis was confined to astrocytes and microglia, but did not occur in neurons. A. Co-immunofluorescence staining of NLRP3 and Iba 1. B. Co-immunofluorescence staining of NLRP3 and GFAP. C. Co-immunofluorescence staining of NLRP3 and Neun.

Additional file 2:. Supplementary figure 2. Colitis increased brain A $\beta$ accumulation as detected by western blots in WT mice but not NLRP3 $\mathrm{KO}$ mice. A. Chemiluminescence imaging of western blots showing that DSS administration did not affect APP expression in either control WT or NLRP3 KO mice, while DSS feeding increased A 40 expression in control WT mice but not NLRP3 KO mice. B. Comparisons of APP/ $\beta$-tubulin (i) and $A \beta 40 / \beta$-tubulin (ii) ratios among control WT, DSS-fed WT, control NLRP3 KO, and DSS-fed NLRP3 KO mice. C. Chemiluminescence imaging of western blots showing that DSS administration increased A $\beta 42$ expression in WT mice but not NLRP3 KO mice. D. Comparisons of A $\beta$ 42/ $\beta$-tubulin ratio among control WT, DSS-fed WT, control NLRP3 KO, and DSS-fed NLRP3 KO mice. Each dataset is expressed as mean \pm SD. ${ }^{* P} \leq$ $0.05 ;{ }^{* *} \mathrm{P} \leq 0.01 ;{ }^{* * *} \mathrm{P} \leq 0.001 ;{ }^{* * *} \mathrm{P} \leq 0.0001 . \mathrm{n}=3$ mice.

\section{Acknowledgements}

We thank the Neurology Department, First Affiliated Hospital of Sun Yat-sen University (Guangdong Provincial Engineering Center for Major Neurological Disease Treatment; Guangdong Provincial Translational Medicine Innovation Platform for Diagnosis and Treatment of Major Neurological Disease) for providing the experimental equipment and Guangdong Laboratory Animals Monitoring Institute for breeding the NLRP3 Knockout mice.

\section{Authors' contributions}

Xiao-fei He, Li-li Li, Wen-biao Xian, Ming-yue Li, Li-ying Zhang, and Jing-hui Xu performed the experiments. Xiao-fei He and Li-li Li drafted the manuscript. Li-ying Zhang and Hai-qing Zheng revised the manuscript. Hai-qing Zheng and Xi-quan Hu conceived and designed the research, and edited and revised the manuscript. The authors read and approved the final manuscript

\section{Funding}

This work was supported by grants from the National Natural Science Foundation of China $(81902285,81871847,81672261,81972151,8157228$, 81873751), the Guangdong Basic and Applied Basic Research Foundation (2019A1515011444, 2017A030313493, 2019A1515011106), the Science and Technology Planning Key Project of Guangzhou, China (201803010119), the Guangzhou Health and Medical Collaborative Innovation Major Projects (201604020009), the Guangdong Provincial Key Laboratory for Diagnosis and Treatment of Major Neurological Diseases (2017B030314103), the Southern China International Cooperation Base for Early Intervention and Functional Rehabilitation of Neurological Diseases (2015B050501003), and the Chinese Postdoctoral Science Foundation (2019M663283, 2020T130742). 


\section{Availability of data and materials}

All data generated or analyzed during this study are included in this published article (and its supplementary information files).

\section{Declarations}

\section{Ethics approval and consent to participate}

The study was approved by the Animal Research Committee, Laboratory Animal Monitoring Institute of Guangdong Province (Committee's reference number: [2013]97).

\section{Consent for publication}

All authors of the manuscript have agreed to publish this article.

\section{Competing interests}

The authors have declared that no competing interests exist.

\section{Author details}

'Department of Rehabilitation Medicine, The Third Affiliated Hospital, Sun Yat-sen University, 600 Tianhe Road, Guangzhou 510630, Guangdong, China. ${ }^{2}$ Department of Neurology, National Key Clinical Department and Key Discipline of Neurology, Guangdong Key Laboratory for Diagnosis and Treatment of Major Neurological diseases, The First Affiliated Hospital, Sun Yat-sen University, Guangzhou 510080, Guangdong, China.

\section{Received: 13 April 2021 Accepted: 21 June 2021}

\section{Published online: 06 July 2021}

\section{References}

1. Heneka MT, Kummer MP, Latz E. Innate immune activation in neurodegenerative disease. Nat Rev Immunol. 2014;14(7):463-77. https://doi. org/10.1038/nri3705.

2. Sochocka M, Donskow-Łysoniewska K, Diniz BS, et al. The gut microbiome alterations and inflammation-driven pathogenesis of Alzheimer's disease-a critical review. Mol Neurobiol. 2019;3(56):1841-51.

3. Hardy J, Selkoe DJ. The amyloid hypothesis of Alzheimer's disease: progress and problems on the road to therapeutics. Science. 2002;297(5580):353-6. https://doi.org/10.1126/science.1072994.

4. Wildsmith KR, Holley M, Savage JC, et al. Evidence for impaired amyloid $\beta$ clearance in Alzheimer's disease. Alzheimers Res Ther. 2013;5(4):33.

5. Iliff JJ, Wang M, Liao Y, et al. A paravascular pathway facilitates CSF flow through the brain parenchyma and the clearance of interstitial solutes, including amyloid $\beta$. Sci Transl Med. 2012;4(147):147ra111.

6. Mesquita SD, Louveau A, Vaccari A, et al. Functional aspects of meningeal lymphatics in ageing and Alzheimer's disease. Nature. 2018;560(7717):18591. https://doi.org/10.1038/s41586-018-0368-8.

7. He XF, Liu DX, Zhang Q, et al. Voluntary exercise promotes glymphatic clearance of amyloid beta and reduces the activation of astrocytes and microglia in aged mice. Front Mol Neurosci. 2017;10:144.

8. Harrison IF, Ismail O, Machhada A, et al. Impaired glymphatic function and clearance of tau in an Alzheimer's disease model. Brain. 2020;143(8):257693.

9. Iliff JJ, Chen MJ, Plog BA, Zeppenfeld DM, Soltero M, Yang L, et al. Impairment of glymphatic pathway function promotes tau pathology after traumatic brain injury. J Neurosci. 2014;34(49):16180-93. https://doi.org/10.1 523/JNEUROSCI.3020-14.2014.

10. Liddelow SA, Guttenplan KA, Clarke LE, et al. Neurotoxic reactive astrocytes are induced by activated microglia. Nature. 2017;541(7638):481-7.

11. Clarke LE, Liddelow SA, Chakraborty C, Münch AE, Heiman M, Barres BA. Normal aging induces A1-like astrocyte reactivity. Proc Natl Acad Sci U S A. 2018;115(8):E1896-905. https://doi.org/10.1073/pnas.1800165115.

12. Bonaz BL, Bernstein CN. Brain-gut interactions in inflammatory bowel disease. Gastroenterology. 2013;144(1):36-49. https://doi.org/10.1053/.j.ga stro.2012.10.003

13. Smith PA. The tantalizing links between gut microbes and the brain. Nature. 2015;526(7573):312-4. https://doi.org/10.1038/526312a.

14. Dodiya HB, Kuntz T, Shaik SM, Baufeld C, Leibowitz J, Zhang X, et al. Sexspecific effects of microbiome perturbations on cerebral $A \beta$ amyloidosis and microglia phenotypes. J Exp Med. 2019;216(7):1542-60. https://doi.org/1 0.1084/jem.20182386.
15. Pellegrini C, Antonioli L, Calderone V, et al. Microbiota-gut-brain axis in health and disease: Is NLRP3 inflammasome at the crossroads of microbiota-gut-brain communications? Prog Neurobiol. 2020;191:101806.

16. Heneka MT, Kummer MP, Stutz A, et al. NLRP3 is activated in Alzheimer's disease and contributes to pathology in APP/PS1 mice. Nature, 2013, 493(7434): 674-678.

17. Martin JC, Beriou G, Josien R. Dextran sulfate sodium (DSS)-induced acute colitis in the rat. Methods Mol Biol. 2016;1371:197-203. https://doi.org/10.1 007/978-1-4939-3139-2_12.

18. Solomon L, Mansor S, Mallon P, et al. The dextran sulphate sodium (DSS) model of colitis: an overview. Comp Clin Pathol. 2010;19(3):235-9.

19. Feng $Y$, He $X$, Luo S, Chen $X$, Long S, Liang F, et al. Chronic colitis induces meninges traffic of gut-derived T cells, unbalances M1 and M2 microglia/ macrophage and increases ischemic brain injury in mice. Brain Res. 2019; 1707:8-17. https://doi.org/10.1016/j.brainres.2018.11.019.

20. Wirtz S, Neufert C, Weigmann B, Neurath MF, et al. Chemically induced mouse models of intestinal inflammation. Nat Protoc. 2007;3(2):541-6.

21. Seibenhener ML, Wooten MC. Use of the open field maze to measure locomotor and anxiety-like behavior in mice. J Vis Exp. 2015(96):e52434.

22. Williams MT, Vorhees CV. Morris water maze: procedures for assessing spatial and related forms of learning and memory. Nat Protoc. 2006;1(2): 848-58.

23. He XF, Lan Y, Zhang Q, Liu DX, Wang Q, Liang FY, et al. Deferoxamine inhibits microglial activation, attenuates blood-brain barrier disruption, rescues dendritic damage, and improves spatial memory in a mouse model of microhemorrhages. J Neurochem. 2016;138(3):436-47. https://doi.org/1 0.1111/jnc.13657.

24. Lueptow LM. Novel object recognition test for the investigation of learning and memory in mice. J Vis Exp. 2017;126:55718.

25. Clark R, Zola S, Squire L. Impaired recognition memory in rats after damage to the Hippocampus. J Neurosci. 2000;23(20):8853-60.

26. Kaufman AC, Salazar SV, Haas LT, et al. Fyn inhibition rescues established memory and synapse loss in Alzheimer mice. Ann Neurol. 2015;77(6):95371.

27. Singh V, Roth S, Llovera G, Sadler R, Garzetti D, Stecher B, et al. Microbiota dysbiosis controls the neuroinflammatory response after stroke. J Neurosci. 2016;36(28):7428-40. https://doi.org/10.1523/JNEUROSCI.1114-16.2016.

28. Louveau A, Smirnov I, Keyes TJ, et al. Structural and functional features of central nervous system lymphatic vessels. Nature. 2015;7560(523):337-41.

29. Xiao $\mathrm{H}$, Lin $\mathrm{C}, \mathrm{Ho} \mathrm{DHH}$, et al. Inhibitory effect of the gallotannin corilagin on dextran sulfate sodium-induced murine ulcerative colitis. J Nat Prod. 2013; 76(11):2120-5.

30. Chen J, Jayachandran M, Zhang W, et al. Dietary supplementation with sea bass (Lateolabrax maculatus) ameliorates ulcerative colitis and inflammation in macrophages through inhibiting Toll-like receptor 4-linked pathways. Int J Mol Sci. 2019;20(12):2907.

31. Jeon $Y D$, Lee $J H$, Lee $Y M$, et al. Puerarin inhibits inflammation and oxidative stress in dextran sulfate sodium-induced colitis mice model. Biomed Pharmacother. 2020;124:109847.

32. Andujar I, Recio MC, Giner RM, et al. Inhibition of ulcerative colitis in mice after oral administration of a polyphenol-enriched cocoa extract is mediated by the inhibition of STAT1 and STAT3 phosphorylation in colon cells. J Agric Food Chem. 2011;59(12):6474-83.

33. Benakis C, Brea D, Caballero S, Faraco G, Moore J, Murphy M, et al. Commensal microbiota affects ischemic stroke outcome by regulating intestinal gammadelta T cells. Nat Med. 2016;22(5):516-23. https://doi.org/1 0.1038/nm.4068.

34. Ambrosini YM, Borcherding D, Kanthasamy A, et al. The gut-brain axis in neurodegenerative diseases and relevance of the canine model: a review. Front Aging Neurosci. 2019;11:130.

35. Zhang $T$, Han $Y$, Wang JY, et al. Comparative epidemiological investigation of Alzheimer's disease and colorectal cancer: the possible role of gastrointestinal conditions in the pathogenesis of AD. Front Aging Neurosci. 2018;10:176.

36. Navabi S, Gorrepati VS, Yadav S, Chintanaboina J, et al. Influences and impact of anxiety and depression in the setting of inflammatory bowel disease. Inflamm Bowel Dis. 2018;11(24):2303-8.

37. Jorm AF. History of depression as a risk factor for dementia: an updated review. Aust N Z J Psychiatry. 2001;35(6):776-81.

38. Evans IEM, Llewellyn DJ, Matthews FE, Woods RT, Brayne C, Clare L. Social isolation, cognitive reserve, and cognition in older people with depression 
and anxiety. Aging Ment Health. 2019;23(12):1691-700. https://doi.org/10.1 080/13607863.2018.1506742.

39. Richard E, Reitz C, Honig LH, et al. Late-life depression, mild cognitive impairment, and dementia. JAMA Neurol, 2013, 70(3):374-82.

40. Ren P, Chen J, Li B, et al. Nrf2 ablation promotes Alzheimer's disease-like pathology in APP/PS1 transgenic mice: the role of neuroinflammation and oxidative stress. Oxidative Med Cell Longev. 2020;2020:3050971.

41. Datta M, Staszewski O, Raschi E, Frosch M, Hagemeyer N, Tay TL, et al, Histone deacetylases 1 and 2 regulate microglia function during development, homeostasis, and neurodegeneration in a context-dependent manner. Immunity. 2018;48(3):514-29. https://doi.org/10.1016/j.immuni.2018. 02.016 .

42. Liu S, Liu Y, Hao W, et al. TLR2 is a primary receptor for Alzheimer's amyloid beta peptide to trigger neuroinflammatory activation. J Immunol. 2012; 188(3):1098-107.

43. Montacute R, Foley K, Forman R, et al. Enhanced susceptibility of triple transgenic Alzheimer's disease (3xTg-AD) mice to acute infection. J Neuroinflammation. 2017;14(1):50.

44. Lin C, Zhao S, Zhu Y, Fan Z, Wang J, Zhang B, et al. Microbiota-gut-brain axis and toll-like receptors in Alzheimer's disease. Comput Struct Biotechnol J. 2019;17:1309-17. https://doi.org/10.1016/j.csbj.2019.09.008.

45. Liu CC, Hu J, Zhao N, Wang J, Wang N, Cirrito JR, et al. Astrocytic LRP1 mediates brain abeta clearance and impacts amyloid deposition. J Neurosci. 2017;37(15):4023-31. https://doi.org/10.1523/JNEUROSCl.3442-16.2017.

46. Zhang Y, Huang R, Cheng M, et al. Gut microbiota from NLRP3-deficient mice ameliorates depressive-like behaviors by regulating astrocyte dysfunction via circHIPK2. Microbiome. 2019;7(1):116.

47. Rasmussen MK, Mestre $\mathrm{H}$, Nedergaard M. The glymphatic pathway in neurological disorders. Lancet Neurol. 2018;17(11):1016-24. https://doi.org/1 0.1016/S1474-4422(18)30318-1.

48. Kress BT, Iliff JJ, Xia M, Wang M, Wei HS, Zeppenfeld D, et al. Impairment of paravascular clearance pathways in the aging brain. Ann Neurol. 2014;76(6): 845-61. https://doi.org/10.1002/ana.24271.

49. Song L, Pei L, Yao S, et al. NLRP3 Inflammasome in neurological diseases, from functions to therapies. Front Cell Neurosci. 2017:11:63.

50. Yazdi AS, Ghoreschi K. The interleukin-1 family. Adv Exp Med Biol. 2016; (941):21-9.

51. Mesquita SD, Fu Z, Kipnis J. The meningeal lymphatic system: a new player in neurophysiology. Neuron. 2018;100(2):375-88. https://doi.org/10.1016/j. neuron.2018.09.022.

52. Schlager $\mathrm{C}$, Korner $\mathrm{H}$, Krueger $\mathrm{M}$, et al. Effector T-cell trafficking between the leptomeninges and the cerebrospinal fluid. Nature. 2016;530(7590):349-53. https://doi.org/10.1038/nature16939.

53. Zhao H, Wan L, Chen Y, Zhang H, Xu Y, Qiu S. FasL incapacitation alleviates $\mathrm{CD} 4+\mathrm{T}$ cells-induced brain injury through remodeling of microglia polarization in mouse ischemic stroke. J Neuroimmunol. 2018;318:36-44. https://doi.org/10.1016/j.jneuroim.2018.01.017.

54. Buckwalter MS, Coleman BS, Buttini M, et al. Increased T cell recruitment to the CNS after amyloid $\beta 1-42$ immunization in Alzheimer's mice overproducing transforming growth factor- $\beta 1$. J Neurosci, 2006, 26(44): 11437-11441.

55. Louveau A, Herz J, Alme MN, et al. CNS lymphatic drainage and neuroinflammation are regulated by meningeal lymphatic vasculature. Nat Neurosci. 2018;21(10):1380-91.

56. Ahn JH, Cho H, Kim JH, et al. Meningeal lymphatic vessels at the skull base drain cerebrospinal fluid. Nature. 2019;572(7767):62-6. https://doi.org/10.103 8/s41586-019-1419-5.

57. Umiker B, Lee HH, Cope J, et al. The NLRP3 inflammasome mediates DSSinduced intestinal inflammation in Nod2 knockout mice. Innate Immun. 2019;25(2):132-43.

58. Yao X, Zhang $C$, Xing $Y$, et al. Remodelling of the gut microbiota by hyperactive NLRP3 induces regulatory T cells to maintain homeostasis. Nat Commun. 2017:8(1):1896.

\section{Publisher's Note}

Springer Nature remains neutral with regard to jurisdictional claims in published maps and institutional affiliations.

Ready to submit your research? Choose BMC and benefit from:

- fast, convenient online submission

- thorough peer review by experienced researchers in your field

- rapid publication on acceptance

- support for research data, including large and complex data types

- gold Open Access which fosters wider collaboration and increased citations

- maximum visibility for your research: over $100 \mathrm{M}$ website views per year

At BMC, research is always in progress.

Learn more biomedcentral.com/submissions 